\title{
Enhancing robustness of noisy qutrit teleportation with Markovian memory
}

RuiQing $X^{1,2}$, Ri-Gui Zhou ${ }^{1,2^{*}}$ (D), YaoChong $\mathrm{Li}^{1,2}$, SheXiang Jiang ${ }^{3}$ and Hou lan ${ }^{4}$

${ }^{*}$ Correspondence:

rgzhou@shmtu.edu.cn

${ }^{1}$ College of Information Engineering, Shanghai Maritime

University, Shanghai, China

${ }^{2}$ Research Center of Intelligent

Information Processing and

Quantum Intelligent Computing,

Shanghai Maritime University,

Shanghai, China

Full list of author information is

available at the end of the article

\section{Springer}

\begin{abstract}
Quantum teleportation is the fundamental communication unit in quantum communication. Here, a three-level system is selected for storing and transmitting quantum information, due to its unique advantages, such as lower cost than a higher-level system and higher capacity and security than a two-level system. It is known that the key procedure for perfect teleportation is the distribution of entanglement through quantum channel. However, amounts of noise existing in the quantum channel may interfere the entangled state, causing the degradation of quantum entanglement. In the physical implementations of quantum communication schemes, noise acting on the carriers of successive transmissions often exhibits some correlations, which is the so called quantum memory channel. In this paper, a memory channel model during the entanglement distribution phase is constructed and the uniform expression of the evolution of a two-qutrit entangled state under different kinds of correlated noise is derived. Finally, Pauli noise and amplitude damping noise as the typical noise source are considered to analyze the influence of memory effects of noise on qutrit teleportation. It is expected to show that three-level teleportation under these two types of channels can generally enhance the robustness to noise if the Markovian correlations of quantum channel are taken into consideration.
\end{abstract}

Keywords: Noise; Markovian memory; Quantum teleportation; Average fidelity

\section{Introduction}

In the past decades, quantum science and technology has been applied in various fields of information science, such as machine learning [11, 21, 23, 31-33, 49] and network communication $[10,28,36,53,54]$. As one of the most important branches in quantum communication, quantum teleportation is always the focus and forefront project in academic researches. With the help of the shared entanglement and one-way classical communication, quantum teleportation in principle allows the faithful transfer of unknown quantum states from one particle to another at a distance, without physical transmission of the object itself.

The noise existed in the quantum channel is one of the main issues for reliable teleportation of the information. The preshared entangled state that interacted with the external environment might evolve into a mixed state during transmission, leading to

(c) The Author(s) 2022. This article is licensed under a Creative Commons Attribution 4.0 International License, which permits use, sharing, adaptation, distribution and reproduction in any medium or format, as long as you give appropriate credit to the original author(s) and the source, provide a link to the Creative Commons licence, and indicate if changes were made. The images or other third party material in this article are included in the article's Creative Commons licence, unless indicated otherwise in a credit line to the material. If material is not included in the article's Creative Commons licence and your intended use is not permitted by statutory regulation or exceeds the permitted use, you will need to obtain permission directly from the copyright holder. To view a copy of this licence, visit http://creativecommons.org/licenses/by/4.0/. 
the loss of quantum information recovered by the output system. The majority of researches on quantum noise are predicated on the assumption of memoryless channel, where the noisy transformations are performed on the sequences of quantum carriers independently and identically $[19,20,51]$. However, the assumption of vanishing correlation or memory in the system-environment interaction is not always justified. With increasing transmission rates in communication channel, for example, successive transmissions happen so rapidly that the environment may retain a memory between individual transmission. The experimental counterparts of memory effects in quantum communication schemes are limited to the direct transmission of each carrier, such as the photons travelling through optical fiber can be affected by the birefringence fluctuations $[5,6]$ whose characteristic time scales are much longer than the temporal separation between consecutive light pulses; information transmission in a spin chain channel where if the chain is not reset after each use of the channel, the first transmission of information state could influence the second [8]; and the Bosonic channel considering the loss of energy en route where the much higher use rate of channel would induce the unwanted overlaps between consecutive input pulses or interfere with the finite relaxation time of the local environment, leading to the memory effects of attenuation [39, 40]. Interestingly, several models of memory channels have shown that the quantum or classical capacity can be enhanced compared to the results of memoryless channels. Therefore, it is worth considering that how to construct a model of quantum memory channel during the entanglement distribution for improving the communication quality of teleportation.

In recent years, quantum memory channels exhibiting Pauli errors or energy dissipation during the entanglement distribution have been theoretically studied in the twodimensional Hilbert space and it has been discovered that the fidelity of teleportation under these two types of memory channels can be gradually improved with the increasing of the degree of memory [34, 35]. Nevertheless, to teleport quantum states of a real particle, such as a single photon, one needs to consider not only the two-level states (polarization), but also those multi-level states $[25,38,50]$. For example, the orbital angular momentum [16, 37], the temporal mode [3, 42], the frequency mode [30], and the spatial mode $[24,45]$ of a single photon are all natural attributes of multi-level states, which are exploited as high-dimensional systems. It is worth mentioning that ref [22] has made an argument that as the dimension of the constituent qudits increases, the cost of maintaining a qudit in a fully entangled state also increases and the optimum cost per Hilbert dimension is attained at the local dimension of $d=3$ ( $d$ is the dimensionality of Hilbert space). Therefore, a fully entangled state prepared by a three-level system can have a lower cost than that prepared by a higher-level system. On the other hand, compared with conventional two-level systems, three-level quantum states in quantum communication possess both higher capacity and noise resilience $[7,27]$. Taken together, it is of great practice significance to analyze the characteristics of qutrit teleportation in noisy environments and investigate whether the noise correlation in quantum channel can improve the communication quality of noisy teleportation.

In this paper, a model of quantum memory channel during the entanglement distribution is constructed for better understanding the action of memory effects of channel on qutrit teleportation. Then based on the model, a common representation of the dynamical evolution of a maximally two-qutrit entangled state through different kinds of 
noise is derived. Here, Pauli errors and energy dissipation in the channel are paid much attention. Once the operators describing various noise are given, the evolution of a maximally qutrit entangled state can be numerically analyzed. In particular, the Kraus operators of describing correlated amplitude damping channel cannot be directly represented like that of correlated Pauli channel [47]. It is creatively derived by solving the Lindblad equation representing the dynamical evolution of the compound system of two three-level V-type atoms. Finally, the communication quality of teleportation is evaluated by average fidelity which is the function of noise intensity and the degree of memory. It is discovered that qutrit teleportation with Pauli channel (except for phase_flip) or amplitude damping channel has some robustness to noise when memory effects of quantum channel are considered.

Overall, our results prove that the communication quality of noisy teleportation in three dimensions can be enhanced to some extent when considering the two consecutive uses of channel with partial memory, which providing a new thought for reliable information transmission with higher capacity and security.

\section{Results}

Quantum teleportation as the fundamental element of quantum communication plays a key role in quantum computing [43] and quantum internet [14, 15, 46, 48]. Noise environment is always the pivotal factor for destroying the quantum properties, notoriously coherence and entanglement. However, noise may be amicable to information transmission when its classical correlations are taken into consideration. Therefore, it is significant to investigate the memory effects of noisy channel that may exist in realistic entanglement distribution for inhibiting the negative effects of noise on teleportation.

\subsection{Dynamical evolution of two-qutrit entangled state under different correlated noise environment}

The channel model for entanglement distribution is constructed under the assumption that the light pulse will be transferred through a lossy photon channel and a noiseless channel to the communication party in sequence. The memory effects of lossy photon channel may emerge when two photons of the entangled photonic state are successively transmitted through the channel with the separation time scale of two consecutive transmissions much lower than the finite relaxation time of local environment interacted with the external reservoir. The diagram of the channel model is shown in Fig. 1.

Now, assume the preshared two-qutrit entangled photonic state is represented by the density matrix $\rho_{Q}$, then the dynamical evolution of state $\rho_{Q}$ transmitted through the lossy memory channel which has been derived in the Method can be written as

$$
\Phi^{\otimes 2}\left(\rho_{Q}\right)=(1-\mu) \sum_{i_{1}, i_{2}} p_{i_{2}} p_{i_{1}} \Phi_{q_{2}}^{\left(i_{2}\right)} \otimes \Phi_{q_{1}}^{\left(i_{1}\right)}\left(\rho_{Q}\right)+\mu \sum_{i_{1}} p_{i_{1}} \Phi_{q_{2}}^{\left(i_{1}\right)} \otimes \Phi_{q_{1}}^{\left(i_{1}\right)}\left(\rho_{Q}\right),
$$

where the parameter $\mu \in[0,1]$ is the degree of memory describing the overlap ratio between two consecutive transmissions of photons. $\Phi_{q_{j}}^{(i)}$ is an arbitrary CPTP map on system state with the corresponding probability $p_{i}$. It is worth noting that the derivation of Eq. (1) is based on two assumptions, one is the Markovian behavior of lossy photon channel that is the action of past channel can only influence the current channel use and is irrelevant 


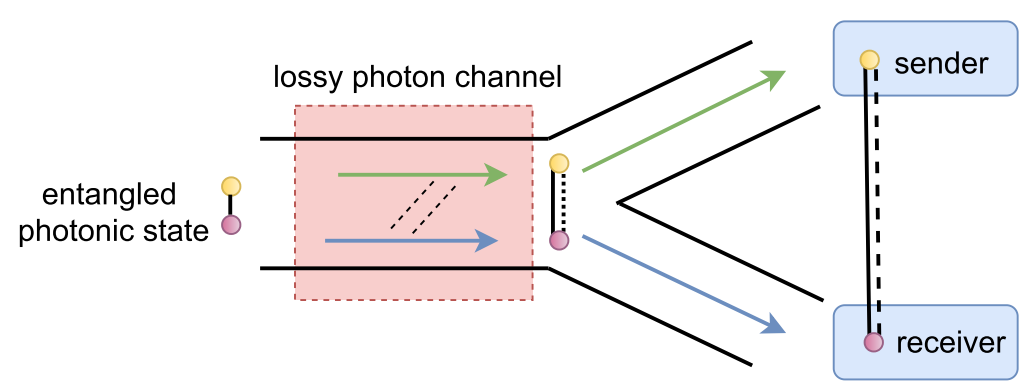

Figure 1 The diagram of the information transmission in the entanglement distribution. The green arrow represents the transmission of the first photon and the blue arrow describes the transmission of the second photon after the separation time $\boldsymbol{\tau}_{s}$. The dotted line is the correlation of noise between two uses of the lossy channel which originates from the much higher rate of consecutive transmission making the local environment retains a memory. The whole transmission process is the entangled photonic state transmitted through lossy memory channel produces a two-photon state exhibiting the coherence and the part of classical correlation and then transmitting the two-photon state through private noiseless channel to the sender and receiver

to the future channel state; the other is assume that two photons of the entangled photonic state will subject to the same local environment with much higher use rates of the lossy channel for determining the mathematical representation of the channel's Markovian behavior. Next, according to the model of quantum memory channel during the entanglement distribution, the evolution of two-qutrit entangled state under the following two typical noise environment is demonstrated for analyzing the influence of Markovian memory channel on qutrit teleportation.

\subsubsection{Pauli noise}

If the lossy photon channel exhibits Pauli errors, the evolution of maximally two-qutrit entangled state transmitted through correlated Pauli channel in three dimensions could be directly characterized by replacing the map $\Phi_{q_{1}\left(q_{2}\right)}^{i_{1}\left(i_{2}\right)}$ with the Weyl operators $U_{n m}=$ $\sum_{l=0}^{2} e^{2 \pi i l n / 3}|l\rangle\langle(l+m) \bmod 3|,\{n, m\} \in\{0,1,2\}$, which is written as

$$
\begin{aligned}
\Phi^{\otimes 2}\left(\rho_{Q}\right)= & (1-\mu) \sum_{\substack{e, f \\
j, k}} p_{e f} p_{j k} U_{e f} \otimes U_{j k} \rho_{Q} U_{e f}^{\dagger} \otimes U_{j k}^{\dagger} \\
& +\mu \sum_{r, s} p_{r s} U_{r s} \otimes U_{r s} \rho_{Q} U_{r s}^{\dagger} \otimes U_{r s}^{\dagger} .
\end{aligned}
$$

The corresponding probability $p_{n m}$ for performing the Weyl operator has defined in ref [41]. It is noticed that different value of $\{n, m\}$ corresponds to different types of Pauli noise, such as trit_flip region, $n=0$ and $m \in\{0,1,2\}$; phase_flip region, $\{n, m\} \in\{0,1,2\}$ and $m=0$; trit_phase flip region, $\{n, m\} \in\{0,1,2\}$ and $(n, m)=(0,0)$; depolarizing noise region, $\{n, m\} \in\{0,1,2\}$.

\subsubsection{Amplitude damping noise}

Amplitude damping channel can be described by a process of energy dissipation of a quantum system to environment. The transition of excitation occurs between excited state and the ground state with a finite probability. Herein, it is assumed that the transitions between two excited states are not allowed. The Kraus operators describing amplitude damping 
noise in three dimensions are concluded in ref $[2,17,18,44]$, which are represented in the standard computational basis:

$$
\begin{aligned}
& E_{0}=|0\rangle\langle 0|+\sqrt{1-P}(|1\rangle\langle 1|+| 2\rangle\langle 2|), \\
& E_{1}=\sqrt{P}|0\rangle\langle 1|, \\
& E_{2}=\sqrt{P}|0\rangle\langle 2|,
\end{aligned}
$$

where $P$ is the magnitude of the energy dissipation. The evolution of two-qutrit maximally entangled state interacted with the correlated amplitude damping can be calculated by the Eq. (1), which is shown as

$$
\Phi^{\otimes 2}\left(\rho_{Q}\right)=(1-\mu) \sum_{i, j} E_{i} \otimes E_{j} \rho_{Q} E_{i}^{\dagger} \otimes E_{j}^{\dagger}+\mu \sum_{k} p_{k} E_{k k} \rho_{Q} E_{k k}^{\dagger} .
$$

Combined Eq. (3) and Eq. (4), it is found that $E_{k k}$ in the correlated term cannot be directly written like the Pauli noise due to the non-unitary property of amplitude damping. Therefore, the Lindblad equation describing quantum Markov channel is introduced. A three-level atomic system in V configuration is the typical case for demonstrating the spontaneous decay of atoms. The Kraus operators $E_{k k}$ can be derived only if we solve the Lindblad equation of the compound system of two three-level atomic systems in V-type. The details of derivation are demonstrated in Methods and the results are given as follows:

$$
\begin{aligned}
& E_{00}=\left[\begin{array}{llll}
\mathbf{I}_{4} & & & \\
& \sqrt{1-P_{1}} & & \\
& & \mathbf{I}_{3} & \\
& & & \sqrt{1-P_{2}}
\end{array}\right]_{9 \times 9} \text {, } \\
& E_{11}=\left[\begin{array}{c|cccc} 
& \sqrt{P_{1}} & 0 & \cdots & 0 \\
& 0 & 0 & \cdots & 0 \\
& \vdots & \vdots & \ddots & \vdots \\
& 0 & 0 & \cdots & 0 \\
\hline \mathbf{O}_{4} & & & &
\end{array}\right]_{9 \times 9} \text {, } \\
& E_{22}=\left[\begin{array}{cccc}
0 & \cdots & 0 & \sqrt{P_{2}} \\
0 & \cdots & 0 & 0 \\
\vdots & \ddots & \vdots & \vdots \\
0 & \cdots & 0 & 0
\end{array}\right]_{9 \times 9}
\end{aligned}
$$

with $P_{1}=1-e^{-\gamma_{10} t}, P_{2}=1-e^{-\gamma_{20} t} . \mathbf{I}_{\mathbf{m}}$ is the identity matrix of $m \times m, \mathbf{O}_{\mathbf{n}}$ is the null matrix of $n \times n$. According to the matrix form of $E_{11}$ and $E_{22}$, we can directly achieve the Kraus operators $E_{1}^{\prime}$ and $E_{2}^{\prime}$ describing the amplitude damping noise in the three-level system, which are described as $E_{1}^{\prime}=\sqrt{P_{1}}|0\rangle\left\langle 1\left|, E_{2}^{\prime}=\sqrt{P_{2}}\right| 0\right\rangle\langle 2|, P_{1}, P_{2}$ are the attenuation coefficients. $E_{0}^{\prime}=|0\rangle\left\langle 0\left|+\sqrt{1-P_{1}}\right| 1\right\rangle\left\langle 1\left|+\sqrt{1-P_{2}}\right| 2\right\rangle\langle 2|$ can be derived with the completeness relation $\sum_{i=0}^{2} E_{i}^{\prime \dagger} E_{i}^{\prime}=I$.

Comparing our results with the Kraus operators of describing amplitude damping noise in Eq. (3), it can be found that they are equivalent if the two attenuation coefficients are assumed in the same value. Clearly, Eq. (3) can be represented as the special case of the Kraus 
operators describing amplitude damping noise in the three-level system, which means the mathematical representation we derived about the correlated amplitude damping noise is general applicably. Finally, putting all known Kraus operators into Eq. (4), the dynamical evolution of entangled photonic state under the lossy memory channel with the loss of photons can be calculated.

\subsection{Qutrit teleportation based on the noisy quantum channel with memory effects}

Quantum information to be teleported cannot be completely recovered from the receiver for the presence of noise in quantum channel. The differences between input state and output state can be quantitatively measured by fidelity [26]. Here, the average fidelity $\langle F\rangle$ is introduced for eliminating the effects of the input state, which is the function of the noise intensity and the degree of memory and can be defined by

$$
\langle F\rangle=\frac{2}{\pi^{2}} \int_{0}^{\frac{\pi}{2}} \int_{0}^{2 \pi} \prod_{i=0}^{1} d \theta_{i} d \phi_{i+1} \sin ^{3-2 i} \theta_{i} \cos \theta_{i} F(\theta, \phi)
$$

the meanings of mathematical symbols are shown in Methods.

According to Eq. (8), the characteristics of qutrit teleportation under correlated Pauli channel and amplitude damping channel are investigated. For the sake of convenience, we stipulate that the name of average fidelity is substituted into fidelity in the following elaboration.

\subsubsection{Transmitting information under correlated Pauli channels}

In the case of the maximally two-qutrit entangled quantum state interacted with uncorrelated Pauli-like noise, the fidelity of qutrit quantum teleportation under trit_flip, phase_flip, trit_phase flip and depolarizing noise is ordinally computed as

$$
\begin{aligned}
& \left\langle F_{T}^{u}\right\rangle=\left\langle F_{P}^{u}\right\rangle=1-\frac{3}{4}\left(2 P-\frac{3}{2} P^{2}\right), \\
& \left\langle F_{T P}^{u}\right\rangle=1-\frac{3}{4}\left(2 P-\frac{5}{4} P^{2}\right), \\
& \left\langle F_{D}^{u}\right\rangle=1-\frac{3}{4}\left(\frac{16}{9} P-\frac{8}{9} P^{2}\right),
\end{aligned}
$$

where $P$ is the noise intensity satisfying $0 \leq P \leq 1$. The relations between fidelity and noise intensity are shown in Fig. 2, from which we can find that taking no account of the value of $P=\frac{2}{3}$, the communication quality of qutrit quantum teleportation under trit-flip noise and phase-flip noise is always superior to classical communication of which fidelity is $\frac{1}{2}$ [4]. In addition, the information transmitted through depolarizing channel causes the greater distortion than phase-flip channel with the noise intensity increasing. 


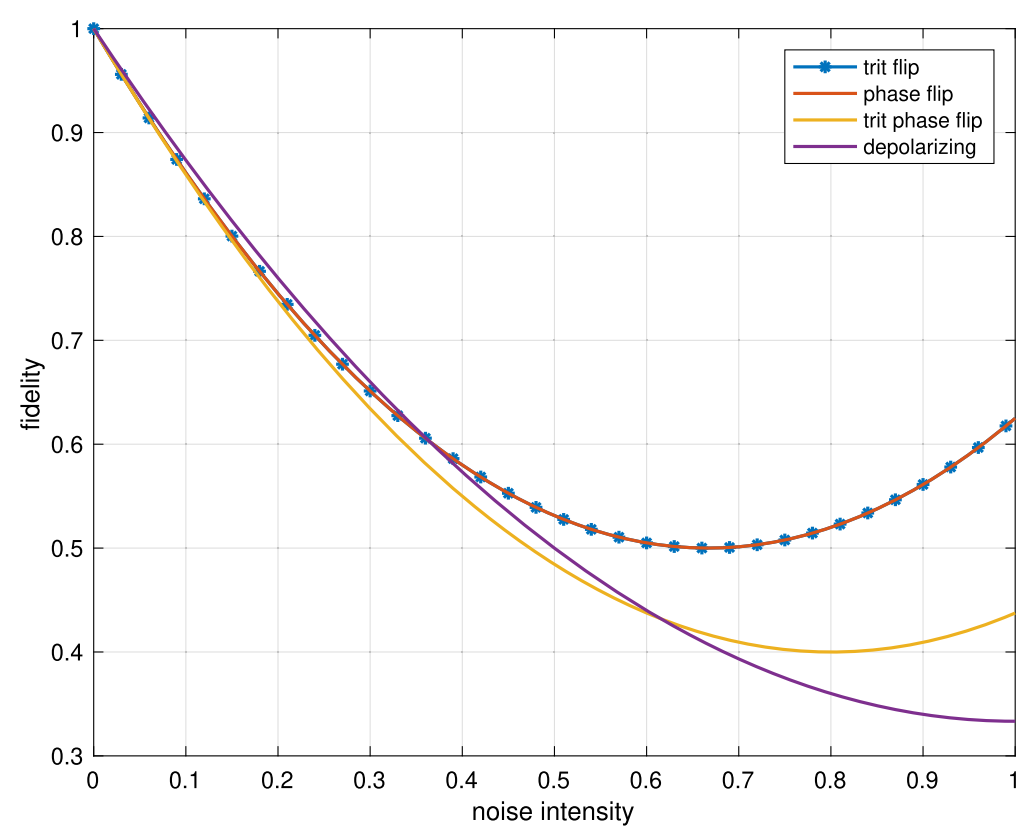

Figure 2 A diagramming drawing of the relations between fidelity and noise intensity of Pauli channels

Considering the interaction of two-qutrit entangled quantum state with correlated Pauli noise, the fidelity of qutrit quantum teleportation is calculated as

$$
\begin{aligned}
& \left\langle F_{T}^{c}\right\rangle=1-\frac{3}{4}(1-\mu)\left(2 P-\frac{3}{2} P^{2}\right), \\
& \left\langle F_{P}^{c}\right\rangle=1-\frac{3}{4}\left(2 P-\frac{3}{2} P^{2}-\mu\left(P-\frac{3}{2} P^{2}\right)\right), \\
& \left\langle F_{T P}^{c}\right\rangle=1-\frac{3}{4}\left(2 P-\frac{5}{4} P^{2}-\mu\left(P-\frac{5}{4} P^{2}\right)\right), \\
& \left\langle F_{D}^{c}\right\rangle=1-\frac{3}{4}\left(\frac{16}{9} P-\frac{8}{9} P^{2}-\mu\left(\frac{10}{9} P-\frac{8}{9} P^{2}\right)\right) .
\end{aligned}
$$

The pictures describing the relations between fidelity, the degree of memory, and noise intensity under the four types of Pauli noise are exhibited in Fig. 3. It is clear that when the preshared quantum state is coupled with correlated trit-flip noise, the fidelity $\left\langle F_{T}^{c}\right\rangle$ will improve to 1 with the increase of the degree of memory no matter what the noise intensity is.

Taking advantage of the memory effects of quantum channel, the fidelity of quantum information that can be reliably teleported through Pauli channel in two-dimensional Hilbert space is greatly enhanced [35]. However, the results of qutrit teleportation under correlated Pauli noise are intuitively not so satisfactory.

Here, we estimate whether the memory effects of quantum channel can improve the transmission quality by numerically analyzing the relationship between noise intensity and the degree of memory on the condition that the communication quality of qutrit quantum teleportation gains an advantage over classical communication. 


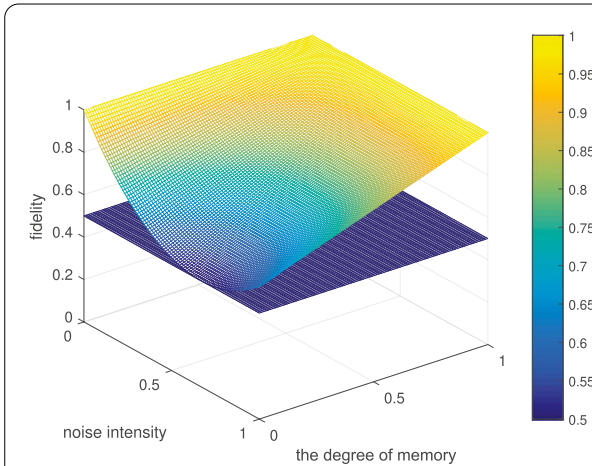

(a)

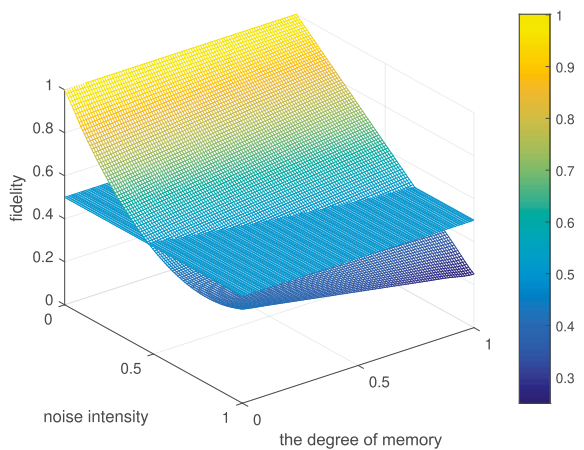

(c)

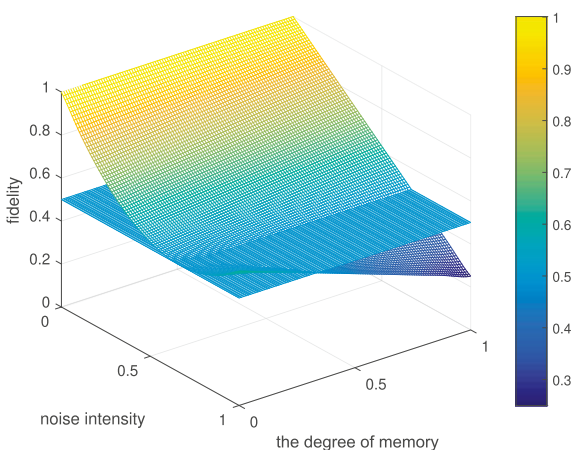

(b)

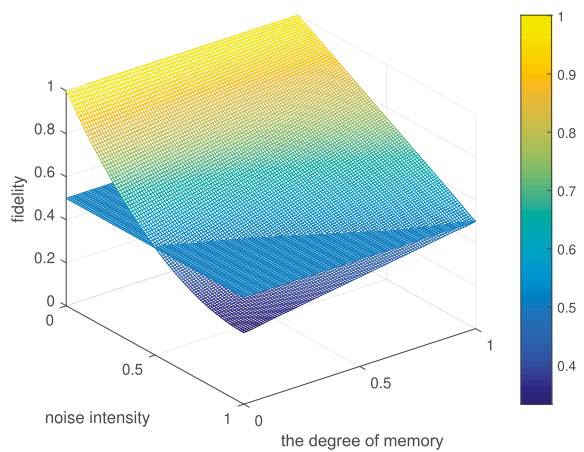

(d)

Figure 3 The relations between fidelity, noise intensity and the degree of memory under trit_flip, phase_flip, trit_phase flip and depolarizing noise are displayed in sequence

For the especial one, trit_flip channel, the inequation connecting noise intensity and the degree of memory is

$$
\mu>1-\frac{1}{3}\left(\frac{1}{P}+\frac{3}{4-3 P}\right), \quad \forall P \in[0,1] .
$$

It is obvious that critical state $1-\frac{1}{3}\left(\frac{1}{P}+\frac{3}{4-3 P}\right)$ is always less than or equal to zero so that the degree of memory always belongs to $(0,1]$ regardless of the noise intensity, which signifies that fidelity of qutrit quantum teleportation through correlated trit_flip channel is constantly higher than that of classical communication with the increase of the degree of memory whatever the noise intensity is.

In the case of correlated phase_flip channel, the relation can be calculated as the following inequations

$$
\begin{aligned}
& \forall \mu \in[0,1], \quad 0 \leq P<\frac{2}{3}, \\
& 0<\mu<1-\frac{2}{3 P}, \quad \frac{2}{3} \leq P \leq 1
\end{aligned}
$$

which means that the fidelity of qutrit quantum teleportation is irrelevant to the correlated coefficient $\mu$ in the condition of $P \in\left[0, \frac{2}{3}\right)$, but when $P$ belongs to the interval $\left[\frac{2}{3}, 1\right]$, the qutrit quantum teleportation would be performed better than classical communication if $\mu$ is less than $1-\frac{2}{3 P}$. Nevertheless, with the degree of memory gradually increasing to 1 , 
the quantum advantages of communication will disappear, which means the teleportation quality under correlated phase_flip channel performs worse than that under the memoryless channel.

For correlated trit_phase flip channel and depolarizing channel, we can derive the critical conditions of the relations between noise intensity and the degree of memory, which can be respectively described as

$$
\begin{aligned}
& \mu_{t r-p h}=1-\frac{2}{3}\left(\frac{1}{P}-\frac{1}{4-5 P}\right), \\
& \mu_{\text {dep }}=1-\frac{3}{5}\left(\frac{1}{P}-\frac{1}{5-4 P}\right) .
\end{aligned}
$$

The constraint graph is drawn in Fig. 4. In order to guarantee the fidelity of qutrit quantum teleportation is greater than $\frac{1}{2}$, the noise intensity and the degree of memory existed in correlated trit_phase flip channel and depolarizing channel must separately satisfy the following criterions

$$
\begin{aligned}
& \left\{\begin{array}{l}
\mu_{t r_{-} p h} \in[0,1], \quad 0 \leq P<0.4734, \\
\mu_{t r-p h}>1-\frac{2}{3}\left(\frac{1}{P}-\frac{1}{4-5 P}\right), \quad 0.4734 \leq P \leq 0.6667,
\end{array}\right. \\
& \left\{\begin{array}{l}
\mu_{d e p} \in[0,1], \quad 0 \leq P<0.5, \\
\mu_{\text {dep }}>1-\frac{3}{5}\left(\frac{1}{P}-\frac{1}{5-4 P}\right), \quad 0.5 \leq P \leq 1 .
\end{array}\right.
\end{aligned}
$$

Combining the constraint graph and the criterion, it can be discovered that the noise intensity can enlarge to a specific threshold with the improvement of the degree of memory in the condition of higher fidelity of qutrit quantum teleportation than classical communication. In the other words, three-level quantum teleportation may possess higher capacity

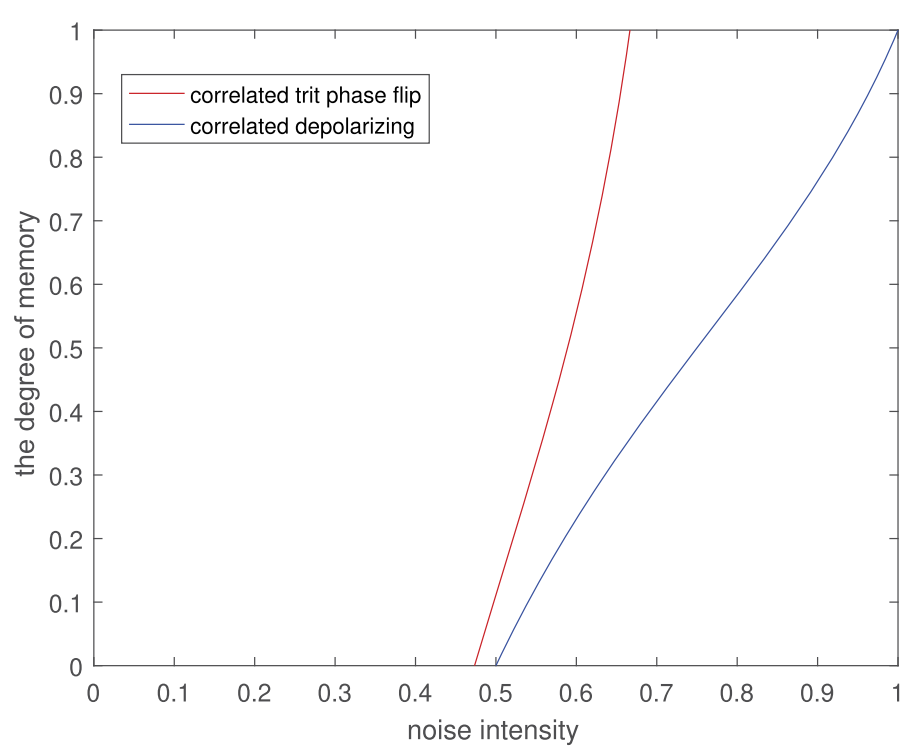

Figure 4 The relations between noise intensity and the degree of memory in correlated trit_phase flip channel and correlated depolarizing channel 
to tolerate trit_phase flip and depolarizing noise when considering the memory effects of quantum channel.

To conclude, distributing entanglement through Pauli channel with Markovian memory can enhance the anti-noise capacity of qutrit teleportation to some extent, except for the phase flip channel.

\subsubsection{Transmitting information under correlated amplitude damping channel}

The fidelity of qutrit teleportation under correlated amplitude damping noise is derived as

$$
\begin{aligned}
\left\langle F_{a m}^{c}\right\rangle= & \frac{2}{3}+\frac{\mu}{6}\left(\sqrt{\left(1-P_{1}\right)\left(1-P_{2}\right)}+\sqrt{1-P_{1}}+\sqrt{1-P_{2}}-1\right) \\
& +\frac{1-\mu}{12}\left(2 P_{1}^{2}+2 P_{2}^{2}+2 P_{1} P_{2}-6 P_{1}-6 P_{2}+4\right) .
\end{aligned}
$$

Taking the case of $\mu=0$, the above formula will become

$$
\left\langle F_{a m}^{u}\right\rangle=\frac{2}{3}+\frac{1}{12}\left[\left(P_{1}-1\right)^{2}+\left(P_{2}-1\right)^{2}+P_{1}^{2}+P_{2}^{2}+\left(2 P_{1}-2\right)\left(P_{2}-1\right)-2 P_{1}-2 P_{2}\right]
$$

which is utilized to describes the influence of uncorrelated amplitude damping noise on teleportation. It is extremely complicated to numerically analyze the criteria that the fidelity of quantum teleportation is higher than that of classical communication. However, the relations between noise intensity $P_{1}, P_{2}$ and fidelity $\left\langle F_{a m}^{c}\right\rangle$ can be simulated with the distinct degree of memory $\mu$, which are shown in Fig. 5.

The relations between fidelity and noise intensity in the condition of $\mu=0, \mu=0.5, \mu=1$ presented in (a), (b), (c) separately possess a general character that the transmission quality will gradually diminish with the increase of noise intensities $P_{1}$ and $P_{2}$. Nevertheless, it is clear that the fidelity of teleportation under amplitude damping channel with Markovian memory can be improved when the values of noise intensities $P_{1}$ and $P_{2}$ are defined.

Investigating the influence of the memory effects of amplitude damping channel on the transmission quality of teleportation for convenience, we assume that the two attenuation coefficients $P_{1}$ and $P_{2}$ are identical to $P$. Then as shown in Fig. 5(d), the fidelity can be enhanced with the increase of the degree of memory when the value of noise intensity is fixed except the case of maximal attenuation. On the other hand, the teleportation can endure more noise with the stronger degree of memory if the fidelity is given. Therefore, taking Markovian memory of amplitude damping channel into consideration is shown to be valuable for noisy teleportation to boost its transmission quality.

\section{Remarks and conclusion}

The realistic communication schemes where temporal rates at which signals are fed into the communication line are too rapid to allow for a resetting of the channel environment can lead to the presence of memory effects. Here, the Markovian model in three-level systems is introduced to construct correlated Pauli channel and amplitude damping channel. The dynamical evolution of the preshared maximally two-qutrit entangled state under Pauli channel and amplitude damping channel with Markovian correlations are derived by Eq. (1) where the operators representing Pauli errors and loss of photons are respectively based on two different kinds of basis, that is a set of Weyl operators $\left\{U_{n m}\right\},\{n, m\} \in\{0,1,2\}$ 


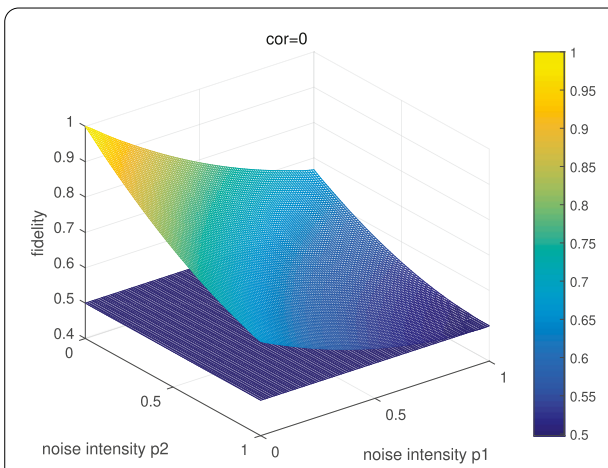

(a)

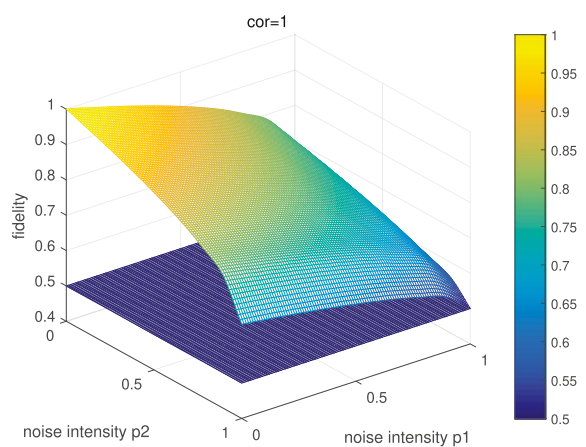

(c)

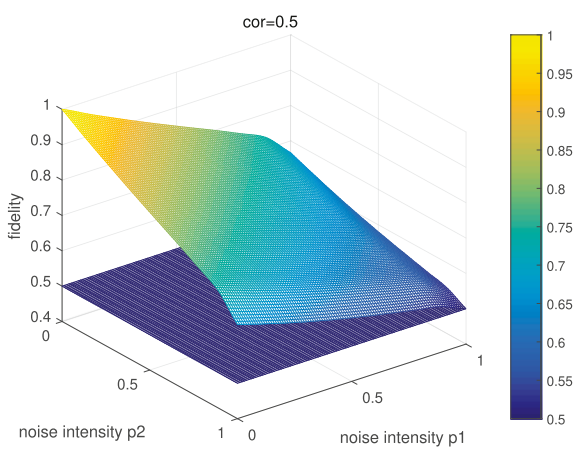

(b)

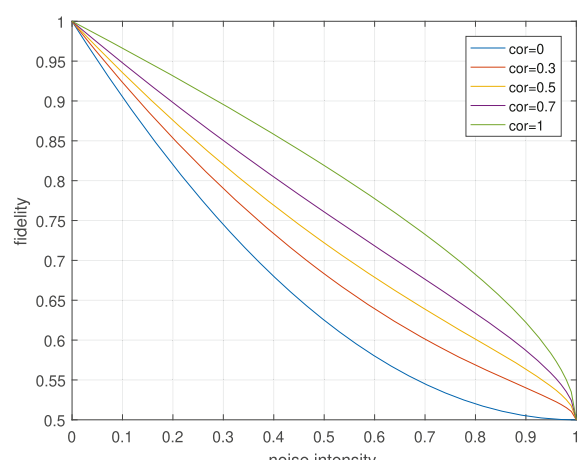

(d)

Figure 5 The effect of the correlated amplitude damping noise on fidelity of qutrit teleportation. The relations between fidelity and noise intensities $P_{1}, P_{2}$ under the circumstances of $\mu=0, \mu=0.5, \mu=1$ are presented in (a), (b), (c) respectively. In the condition of the equivalence of noise intensities $P_{1}$ and $P_{2}$, the connections between the fidelity of teleportation and noise intensity with different values of the degree of memory are established in (d)

and the standard computational basis $\{0,1,2\}$. Particularly, the mathematical representation of describing correlated amplitude damping noise is creatively solved by being connected with the Lindblad equation of the spontaneous emission of a three-level atom in the $\mathrm{V}$ configuration, where the transition between two excited states is dipole forbidden.

According to the mechanism of standard quantum teleportation, the relations between noise intensity and the degree of memory under correlated Pauli channel are numerically analyzed on the condition that teleportation gives the quantum advantages over classical communication. Compared with the teleportation under uncorrelated Pauli channel except for phase_flip channel, the remaining three types of noisy teleportation with Markovian memory are capable to tolerate stronger noise. Considering the phase_flip modeling the erosion of quantum information without loss of energy is immune to the Markovian behavior of quantum channel, it is a method to employ error correction to mitigate its noise on entanglement distribution in the next stage.

In the case of the preshared two-qutrit entangled state interacted with correlated amplitude damping noise, the relations between fidelity and noise intensity can be constructed once the exact value of the degree of memory is given. It is expected that teleportation can withstand stronger noise with the increase of the degree of memory if the fidelity is given in a valid value. 
Generally speaking, it is proved that qutrit teleportation can achieve stronger robustness to noise when taking account of Markovian memory into quantum channel. We are looking forwards to apply this method to actual quantum information processing in the future.

\section{Methods}

\subsection{The model of quantum memory channel during entanglement distribution}

In this paper, the quantum channel is modeled as the combination of two classes of channels, that is a public channel and two private channels. The diagram of this simple channel model is depicted in Fig. 1. The public channel is assumed to be a lossy photon channel where the two photons consisting of entangled photonic state are transmitted in sequence with the separation time scale of two photons much lower than the finite relaxation time of the channel environment. Two private channels are assumed to be ideally noiseless channels that act on the two photons independently and identically.

Although two types of channels are presented, only the lossy photon channel affects the quality of information transmission in the entanglement distribution. Much lower separation time of two photons transmitted through the lossy optical medium could suppress the finite relaxation time of local environment interacted with the external reservoir, leading to the output of the second input photon is affected by both input two photons. It is mentioned that such effects exhibit some classical correlations, not the quantum coherence. To account for such effects, a common memory system $M$ is introduced. Therefore, the corresponding representation of the quantum memory channel can be described as the interaction of the carrier $\rho_{Q}$ with a common memory system $M$ and local environment $e_{i}$, that is

$$
\Phi^{\otimes 2}\left(\rho_{Q}\right)=\operatorname{Tr}_{M E}\left[U_{q_{2} M e_{2}} U_{q_{1} M e_{1}}\left(\rho_{Q} \otimes w_{M} \otimes w_{E}^{\otimes 2}\right) U_{q_{1} M e_{1}}^{\dagger} U_{q_{2} M e_{2}}^{\dagger}\right]
$$

where $\Phi$ is a completely positive and trace-preserving map (CPTP) that transforms states of the sender's end of the channel into states on the receiver's end, $\rho_{Q}, w_{M}$ and $w_{E}$ are entangled photonic state, memory state and local environment state respectively. $U_{q_{i} M e_{i}}$ is the unitary operation that each input photon couples to the memory system and local environment. The graphical sketch of the Eq. (20) is shown in Fig. 6. It is intuitive that each channel use is directly or indirectly affected by the previous one which means the correlated source of noise is presented in the successive uses of channel.

According to existing researches [12, 29], the Eq. (20) can be expressed as the concatenation of 2 CPTP maps, that is

$$
\Phi^{\otimes 2}\left(\rho_{Q}\right)=\operatorname{Tr}_{M}\left[\Phi_{q_{2} M} \circ \Phi_{q_{1} M}\left(\rho_{Q} \otimes w_{M}\right)\right]
$$

where single CPTP map $\Phi_{q_{i} M}$ describing the interaction of a particle of the entangled state with memory system through the unitary dilation, is shown as

$$
I \circ \Phi_{q_{i} M}\left(\rho_{Q} \otimes w_{M}\right)=I \circ \operatorname{Tr}_{e_{i}}\left[U_{q_{i} M e_{i}}\left(\rho_{Q} \otimes w_{M} \otimes w_{e_{i}}\right) U_{q_{i} M e_{i}}^{\dagger}\right]
$$

With the assumption that the action of past channel can only affect that of the current channel and is irrelevant to the future channel state, exhibiting the behavior of Markov 


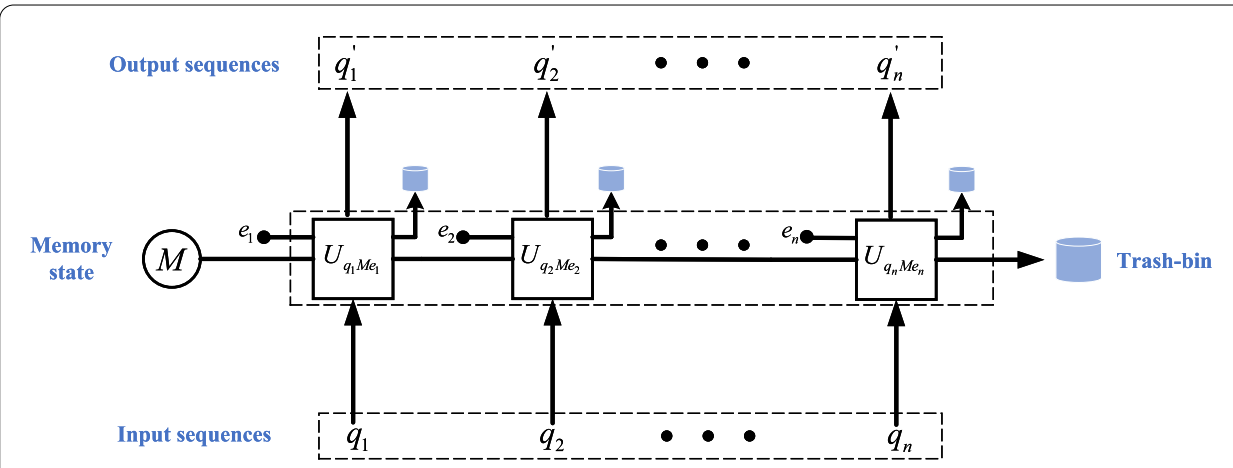

Figure 6 Pictorial representation of the model of memory quantum channel with the $n$ input sequences

chain, the Eq. (21) can be transformed into the following formula

$$
\Phi^{\otimes 2}\left(\rho_{Q}\right)=\sum_{i_{1}, i_{2}} p_{i_{2} \mid i_{1}} p_{i_{1}} \Phi_{q_{2}}^{\left(i_{2}\right)} \otimes \Phi_{q_{1}}^{\left(i_{1}\right)}\left(\rho_{Q}\right)
$$

where $\left\{\Phi_{q_{j}}^{(i)}\right\}_{i}$ is a set of CPTP maps operating on the $j$ th carrier, $p_{i_{1}}$ is the initial probability distribution and $p_{i_{2} \mid i_{1}}$ is a conditional probability.

Furthermore, based on the assumption that the two light pulses will experience the same local environment if the transmission rate of light pulse is much higher than the inverse of the relaxation time of local environment coupled to the external reservoir, the conditional probability $p_{i_{2} \mid i_{1}}$ representing the effect of action of past channel on the current channel can be written as

$$
p_{i_{2} \mid i_{1}}=(1-\mu) p_{i_{2}}+\mu \delta_{i_{1}, i_{1}}
$$

where $\mu \in[0,1]$ is the degree of memory. It is noted that $\mu=0$ means the lossy channel is memoryless and $\mu=1$ means a perfect memory channel where no input information will be revealed to local environment.

Combined with the condition probability described in Eq. (24), Eq. (23) can be rewritten as

$$
\Phi^{\otimes 2}\left(\rho_{Q}\right)=(1-\mu) \sum_{i_{1}, i_{2}} p_{i_{2}} p_{i_{1}} \Phi_{q_{2}}^{\left(i_{2}\right)} \otimes \Phi_{q_{1}}^{\left(i_{1}\right)}\left(\rho_{Q}\right)+\mu \sum_{i_{1}} p_{i_{1}} \Phi_{q_{2}}^{\left(i_{1}\right)} \otimes \Phi_{q_{1}}^{\left(i_{1}\right)}\left(\rho_{Q}\right)
$$

Therefore, the dynamical evolution of two-qutrit entangled state under Pauli noise and amplitude damping noise with Markovian memory can be characterized by Eq. (25) when the operators of describing both noises are presented.

\subsection{The Kraus operators for describing the correlated amplitude damping channel}

The time evolution of a three-level atom in the $\mathrm{V}$ configuration is given by the master equation $\dot{\rho}=-i[H, \rho]+\Lambda \rho[1]$, in which $\rho$ is the density matrix of the three-level atom, $H$ describes the Hamiltonian of the total system, $-i[H, \rho]$ represents the Hamiltonian part 
which is a unitary operation, the damping term $\Lambda \rho$ describing the atom decay by the emission of a photon is defined as

$$
\Lambda \rho=\frac{\gamma_{20}}{2}\left(2 \sigma_{02} \rho \sigma_{20}-\sigma_{22} \rho-\rho \sigma_{22}\right)+\frac{\gamma_{10}}{2}\left(2 \sigma_{01} \rho \sigma_{10}-\sigma_{11} \rho-\rho \sigma_{11}\right),
$$

where $\gamma_{10}$ and $\gamma_{20}$ are the two different spontaneous decay constants that the atom transits from excited states $|1\rangle$ and $|2\rangle$ to ground state $|0\rangle$ respectively. $\sigma_{i j}$ is an atomic transition operator taking an atom from level $|i\rangle$ to $|j\rangle$, which is defined as $\sigma_{i j}=|i\rangle\langle j|$. As amplitude damping channel is used to describe the phenomenon of energy dissipation, it is obvious that the dynamical evolution of a qutrit state under amplitude damping channel can be represented by the Lindblad equation $\dot{\rho}=\Lambda \rho$.

For characterizing the Markovian correlation of the interaction of a compound system $\rho^{c}$ composed of two qutrit states with correlated amplitude damping noise, it is necessary to calculate the Lindblad equation of the two-qutrit state, which is $\dot{\rho}^{c}=\Lambda^{c} \rho^{c}$. The damping term is written as

$$
\Lambda^{c} \rho^{c}=\frac{\gamma_{20}}{2}\left(2 A_{02} \rho^{c} A_{20}-A_{22} \rho^{c}-\rho^{c} A_{22}\right)+\frac{\gamma_{10}}{2}\left(2 A_{01} \rho^{c} A_{10}-A_{11} \rho^{c}-\rho^{c} A_{11}\right),
$$

where $A_{i j}=\sigma_{i j} \otimes \sigma_{i j}$. The method to solve this equation is making use of a special basis of left, $\left\{L_{i}\right\}$, and right, $\left\{R_{i}\right\}$, damping eigenoperators for a Lindblad superoperator $\tilde{\Lambda}$ [13]. It is assumed that density matrix of the three-level compound system is defined as

$$
\rho^{c}=\left[\begin{array}{lllllllll}
\rho_{00} & \rho_{01} & \rho_{02} & \rho_{10} & \rho_{11} & \rho_{12} & \rho_{20} & \rho_{21} & \rho_{22} \\
\rho_{03} & \rho_{04} & \rho_{05} & \rho_{13} & \rho_{14} & \rho_{15} & \rho_{23} & \rho_{24} & \rho_{25} \\
\rho_{06} & \rho_{07} & \rho_{08} & \rho_{16} & \rho_{17} & \rho_{18} & \rho_{26} & \rho_{27} & \rho_{28} \\
\rho_{30} & \rho_{31} & \rho_{32} & \rho_{40} & \rho_{41} & \rho_{42} & \rho_{50} & \rho_{51} & \rho_{52} \\
\rho_{33} & \rho_{34} & \rho_{35} & \rho_{43} & \rho_{44} & \rho_{45} & \rho_{53} & \rho_{54} & \rho_{55} \\
\rho_{36} & \rho_{37} & \rho_{38} & \rho_{46} & \rho_{47} & \rho_{48} & \rho_{56} & \rho_{57} & \rho_{58} \\
\rho_{60} & \rho_{61} & \rho_{62} & \rho_{70} & \rho_{71} & \rho_{72} & \rho_{80} & \rho_{81} & \rho_{82} \\
\rho_{63} & \rho_{64} & \rho_{65} & \rho_{73} & \rho_{74} & \rho_{75} & \rho_{83} & \rho_{84} & \rho_{85} \\
\rho_{66} & \rho_{67} & \rho_{68} & \rho_{76} & \rho_{77} & \rho_{78} & \rho_{86} & \rho_{87} & \rho_{88}
\end{array}\right] .
$$

After converting density matrix $\rho^{c}$ in Hilbert space into Fock-Liouville space [41], the Lindblad superoperator $\tilde{\Lambda}$ is derived as a matrix of $81 \times 81$ which is related to $\gamma_{20}$ and $\gamma_{10}$. Then according to the formula $\tilde{\Lambda} R_{i}=\lambda_{i} R_{i}$, the symbols $\lambda_{i}$ for eigenvalues and $R_{i}$ for right eigenstates are able to be calculated. Here, the values of $\lambda_{i}$ are displayed:

$$
\lambda=(0)_{49},\left(-\frac{\gamma_{10}}{2}\right)_{14},\left(-\frac{\gamma_{20}}{2}\right)_{14},\left(-\frac{\gamma_{10}}{2}-\frac{\gamma_{20}}{2}\right)_{2},-\gamma_{10},-\gamma_{20} .
$$

It is worth noting that the subscripts of eigenvalues indicates the frequency of the appearance of each eigenvalue and two eigenvalues without subscripts means they both appear once. Besides, the left eigenvectors $L_{i}$ as the duals of right eigenvectors can be achieved on the condition that $\operatorname{Tr}\left(L_{i} R_{j}\right)=\delta_{i j}$. Eventually, the dynamical evolution of the threelevel compound systems $\rho^{c}$ can be calculated in accordance with the formula $\Phi\left(\rho^{c}\right)=$ 
$\sum_{i} \operatorname{tr}\left(L_{i} \rho^{c}\right) \exp \left(\lambda_{i} t\right) R_{i}$, which is shown as follows

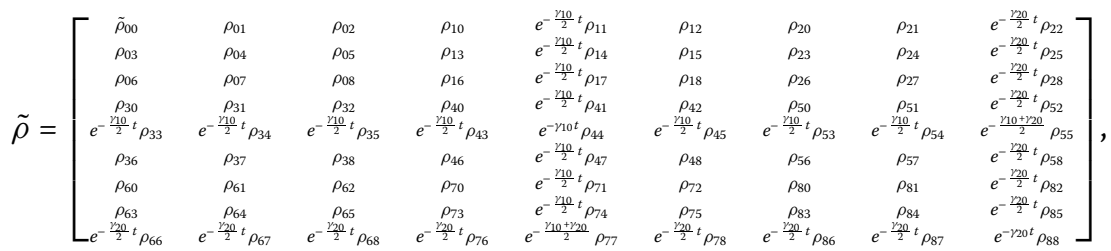

where $\tilde{\rho}_{00}=\rho_{00}+\left(1-e^{-\gamma_{10} t}\right) \rho_{44}+\left(1-e^{-\gamma_{20} t}\right) \rho_{88}$.

As mentioned above, $\sum_{k} E_{k k} \rho_{\mathrm{Q}} E_{k k}$ represents the correlated part of the evolution of twoqutrit entangled state $\rho_{Q}$ under amplitude damping channel with Markovian memory, therefore it is apparent that the Kraus operator $E_{k k}$ can be achieved on the condition that $\sum_{i} \operatorname{tr}\left(L_{i} \rho^{c}\right) \exp \left(\lambda_{i} t\right) R_{i}=\sum_{k} E_{k k} \rho^{c} E_{k k}$, which has been introduced in ref [52]. Combined Eq. (28) and Eq. (30), the operators $E_{00}, E_{11}$ and $E_{22}$ can be finally obtained.

\subsection{Noisy quantum teleportation protocol in three dimensions}

In what follows the standard quantum teleportation in three dimensions is presented.

Step 1: The qutrit state to be transmitted from Alice to Bob is defined as

$$
|\psi\rangle_{i n}=\sum_{i=0}^{2} \alpha_{i}|i\rangle=\cos \theta_{0}|0\rangle+\sin \theta_{0} \cos \theta_{1} e^{i \phi_{1}}|1\rangle+\sin \theta_{0} \sin \theta_{1} e^{i \phi_{2}}|2\rangle,
$$

where $0<\theta_{i} \leq \frac{\pi}{2}$ and $0<\phi_{j} \leq 2 \pi$.

Step 2: Suppose a prior shared maximally entangled state among Alice and Bob is $|\Phi\rangle_{A B}=\frac{1}{\sqrt{3}} \sum_{j=0}^{2}|j\rangle_{A} \otimes|j\rangle_{B}$. After distributing the two particles $A$ and $B$ of the pure state $|\Phi\rangle_{A B}$ through quantum memory channel to communication parties Alice and Bob respectively, the two-qutrit mixed entangled state $M$ can be obtained with Eq. (1).

Step 3: Taking advantage of entanglement, Alice performs a joint measurement on her particles and the corresponding measurement basis [9] is $\left|\phi_{n m}\right\rangle=\frac{1}{\sqrt{3}} \sum_{k=0}^{2} e^{2 \pi i k n / 3}|k\rangle \otimes$ $|(k+m) \bmod 3\rangle$ with $\{n, m\} \in\{0,1,2\}$. Once learning from Alice that she has received the measurement result $(n, m)$, Bob performs a corresponding unitary transformation $U_{n m}=\sum_{l=0}^{2} e^{2 \pi i \ln / 3}|l\rangle\langle(l+m) \bmod 3|$ on his previously entangled particle (particle B) for recovering the information carried by the initial state $|\psi\rangle_{i n}$. Then, the final state of Bob's particle can be written in $\rho_{n m}=U_{n m} \operatorname{Tr}_{A}\left[\left|\phi_{n m}\right\rangle\left\langle\phi_{n m}\right| \otimes I_{B}\left(|\psi\rangle_{i n}\langle\psi| \otimes M\right)\right] U_{n m}^{\dagger}$.

Step 4: Quantum information is inevitably distorted by the noisy quantum channel, the fidelity utilized to estimate the feasibility of the protocol is described as $F_{n m}(\theta, \phi)=$ $\left\langle\psi\left|\rho_{n m}\right| \psi\right\rangle / \operatorname{Tr} \rho_{n m}$. It is worth noting that $\operatorname{Tr}\left(\rho_{n m}\right)^{-1}$ referred as the normalization factor of fidelity is equal to the probability that the result of measurement is $(n, m)$. Taking all possible teleported $\rho_{n m}$ into consideration, the mean fidelity is estimated by $F(\theta, \phi)=$ $\sum_{n m} F_{n m}(\theta, \phi)$.

In order to analyze the effect of noise on the fidelity of qutrit teleportation, it is necessary to construct relations between fidelity and noise intensity without regard to the probability amplitude of the initial state. Therefore, the average fidelity calculated by double integrals of the mean fidelity is eventually chosen to evaluate the transmission quality of quantum teleportation protocol [41], which is written in Eq. (8). 
Acknowledgements

No applicable.

\section{Funding}

This work is supported by the Shanghai Science and Technology Project in 2020 under Grant No.20040501500; FDCT of Macau under Grant No. 0130/2019/A3; University of Macau under Grant No. MYRG2018-00088-IAPME; National Natural Science Foundation of China under Grant No. 11404415

\section{Availability of data and materials}

No applicable.

\section{Declarations}

\section{Competing interests}

The authors declare that they have no competing interests.

\section{Authors' contributions}

$\mathrm{R}-\mathrm{QX}$ is concentrated on writing the article and the mathematical derivation of the Lindblad equation; R-GZ makes contributions to concluding a large number of literature about high-dimensional quantum teleportation; Y-CL contributes to the simulation of the theoretical results; S-XJ participates in the discussion about the solution of the Lindblad equation of two-qutrit compound system and $\mathrm{HI}$ polishes the language expression of the article and corrects some spelling and grammar errors. All authors read and approved the final manuscript.

\section{Author details}

${ }^{1}$ College of Information Engineering, Shanghai Maritime University, Shanghai, China. ${ }^{2}$ Research Center of Intelligent Information Processing and Quantum Intelligent Computing, Shanghai Maritime University, Shanghai, China. ${ }^{3}$ School of Computer Science and Engineering, Anhui University of Science and Technology, Anhui, China. ${ }^{4}$ Institute of Applied Physics and Materials Engineering FST, University of Macau, Macau, China.

\section{Publisher's Note}

Springer Nature remains neutral with regard to jurisdictional claims in published maps and institutional affiliations.

Received: 10 May 2021 Accepted: 28 January 2022 Published online: 10 February 2022

\section{References}

1. Ali M. Distillability sudden death in qutrit-qutrit systems under amplitude damping. J Phys B, At Mol Opt Phys. 2010;43(4):45504.

2. Ann K, Jaeger G. Finite-time destruction of entanglement and non-locality by environmental influences. Found Phys. 2009:39(7):790-828.

3. Ansari V, Donohue JM, Brecht B, Silberhorn C. Tailoring nonlinear processes for quantum optics with pulsed temporal-mode encodings. Optica. 2018;5(5):534-50.

4. Badzia P, Horodecki M, Horodecki P, Horodecki R. Local environment can enhance fidelity of quantum teleportation Phys Rev A. 2000;62(1):12311.

5. Ball J, Dragan A, Banaszek K. Exploiting entanglement in communication channels with correlated noise. Phys Rev A. 2004;69(4):42324.

6. Banaszek K, Dragan A, Wasilewski W, Radzewicz C. Experimental demonstration of entanglement-enhanced classical communication over a quantum channel with correlated noise. Phys Rev Lett. 2004;92(25):257901.

7. Bartůšková L, Černoch A, Filip R, Fiurášek J, Soubusta J, Dušek M. Optical implementation of the encoding of two qubits to a single qutrit. Phys Rev A. 2006;74:022325. https://ink.aps.org/doi/10.1 103/PhysRevA.74.022325.

8. Bayat A, Burgarth D, Mancini S, Bose S. Memory effects in spin-chain channels for information transmission. Phys Rev A. 2008;77:050306. https://link.aps.org/doi/10.1103/PhysRevA.77.050306.

9. Bennett CH, Brassard G, Crépeau C, Jozsa R, Peres A, Wootters WK. Teleporting an unknown quantum state via dual classical and Einstein-Podolsky-Rosen channels. Phys Rev Lett. 1993;70(13):1895-9.

10. Bennett CH, DiVincenzo DP, Shor PW, Smolin JA, Terhal BM, Wootters WK. Remote state preparation. Phys Rev Lett. 2001;87(7):77902.

11. Biamonte J, Wittek P, Pancotti N, Rebentrost P, Wiebe N, Lloyd S. Quantum machine learning. Nature. 2017;549(7671):195-202.

12. Bowen G, Mancini S. Quantum channels with a finite memory. Phys Rev A. 2004;69:012306. https://link.aps.org/doi/10.1103/PhysRevA.69.012306.

13. Briegel HJ, Englert BG. Quantum optical master equations: the use of damping bases. Phys Rev A. 1993:47(4):3311-29.

14. Cacciapuoti AS, Caleffi M, Van Meter R, Hanzo L. When entanglement meets classical communications: quantum teleportation for the quantum Internet. IEEE Trans Commun. 2020;68(6):3808-33.

15. Caleffi M, Cacciapuoti AS. Quantum switch for the quantum Internet: noiseless communications through noisy channels. IEEE J Sel Areas Commun. 2020;38(3):575-88.

16. Dada AC, Leach J, Buller GS, Padgett MJ, Andersson E. Experimental high-dimensional two-photon entanglement and violations of generalized Bell inequalities. Nat Phys. 2011;7(9):677-80.

17. Dutta A, Ryu J, Laskowski W, Żukowski M. Entanglement criteria for noise resistance of two-qudit states. Phys Lett A. 2016;380(27):2191-9.

18. Fonseca A. High-dimensional quantum teleportation under noisy environments. Phys Rev A. 2019:100(6):62311.

19. Fortes R, Rigolin G. Fighting noise with noise in realistic quantum teleportation. Phys Rev A. 2015;92(1):12338. 
20. Fortes R, Rigolin G. Probabilistic quantum teleportation in the presence of noise. Phys Rev A. 2016;93(6):62330.

21. Grant E, Benedetti M, Cao S, Hallam A, Lockhart J, Stojevic V et al. Hierarchical quantum classifiers. npj Quantum Inf. 2018:4(1):65

22. Greentree AD, Schirmer SG, Green F, Hollenberg LCL, Hamilton AR, Clark RG. Maximizing the Hilbert space for a finite number of distinguishable quantum states. Phys Rev Lett. 2004;92(9):97901.

23. Havliček V, Córcoles AD, Temme K, Harrow AW, Kandala A, Chow JM et al. Supervised learning with quantum-enhanced feature spaces. Nature. 2019;567(7747):209-12.

24. Hu XM, Chen JS, Liu BH, Guo Y, Huang YF, Zhou ZQ et al. Experimental test of compatibility-loophole-free contextuality with spatially separated entangled qutrits. Phys Rev Lett. 2016;117(17):170403.

25. Hu XM, Zhang C, Liu BH, Cai Y, Ye XJ, Guo Y et al. Experimental high-dimensional quantum teleportation. Phys Rev Lett. 2020;125(23):230501.

26. Jozsa R. Fidelity for mixed quantum states. J Mod Opt. 1994;41 (12):2315-23.

27. Kaszlikowski D, Oi DKL, Christandl M, Chang K, Ekert A, Kwek LC et al. Quantum cryptography based on qutrit Bell inequalities. Phys Rev A. 2003;67(1):12310.

28. Kimble HJ. The quantum Internet. Nature. 2008:453(7198):1023-30.

29. Kretschmann D, Werner RF. Quantum channels with memory. Phys Rev A. 2005;72:062323. https://link.aps.org/doi/10.1103/PhysRevA.72.062323.

30. Kues M, Reimer C, Roztocki P, Cortés LR, Sciara S, Wetzel B et al. On-chip generation of high-dimensional entangled quantum states and their coherent control. Nature. 2017:546(7660):622-6.

31. Li Y, Zhou RG, Xu R, Hu W, Fan P. Quantum algorithm for the nonlinear dimensionality reduction with arbitrary kernel. Quantum Sci Technol. 2021;6(1):14001.

32. Li Y, Zhou RG, Xu R, Luo J, Hu W. A quantum deep convolutional neural network for image recognition. Quantum Sci Technol. 2020;5(4):44003.

33. Li Y, Zhou RG, XU R, Luo J, Jiang S. A quantum mechanics-based framework for EEG signal feature extraction and classification. In: IEEE transactions on emerging topics in computing. 2020. p. 1.

34. Li YL, Zu CJ, Wei DM. Enhance quantum teleportation under correlated amplitude damping decoherence by weak measurement and quantum measurement reversal. Quantum Inf Process. 2018;18(1):2.

35. Li YL, Zu CJ, Wei DM, Wang CM. Correlated effects in pauli channels for quantum teleportation. Int J Theor Phys. 2019;58(4):1350-8

36. Liao SK, Cai WQ, Liu WY, Zhang L, Li Y, Ren JG et al. Satellite-to-ground quantum key distribution. Nature. 2017;549(7670):43-7.

37. Liu S, Lou Y, Jing J. Orbital angular momentum multiplexed deterministic all-optical quantum teleportation. Nat Commun. 2020;11(1):3875.

38. Luo YH, Zhong HS, Erhard M, Wang XL, Peng LC, Krenn M et al. Quantum teleportation in high dimensions. Phys Rev Lett. 2019:123(7):70505

39. Lupo C, Giovannetti V, Mancini S. Capacities of lossy bosonic memory channels. Phys Rev Lett. 2010;104:030501. https://link.aps.org/doi/10.1103/PhysRevLett.104.030501

40. Lupo C, Giovannetti V, Mancini S. Memory effects in attenuation and amplification quantum processes. Phys Rev A. 2010;82:032312. https://link.aps.org/doi/10.1103/PhysRevA.82.032312.

41. Manzano D. A short introduction to the Lindblad master equation. AIP Adv. 2020;10(2):25106

42. Martin A, Guerreiro T, Tiranov A, Designolle S, Fröwis F, Brunner N et al. Quantifying photonic high-dimensional entanglement. Phys Rev Lett. 2017;118(11):110501.

43. Nielsen MA, Chuang IL. Quantum computation and quantum information. 2010

44. Ramzan M. Three-player quantum Kolkata restaurant problem under decoherence. Quantum Inf Process. 2013;12(1):577-86.

45. Schaeff C, Polster R, Huber M, Ramelow S, Zeilinger A. Experimental access to higher-dimensional entangled quantum systems using integrated optics. Optica. 2015;2(6):523-9.

46. Sun QC, Mao YL, Chen SJ, Zhang W, Jiang YF, Zhang YB et al. Quantum teleportation with independent sources and prior entanglement distribution over a network. Nat Photonics. 2016;10(10):671-5.

47. ur Rehman J, Farooq A, Shin H. Discrete Weyl channels with Markovian memory. IEEE J Sel Areas Commun. 2020:38(3):413-26.

48. Valivarthi R, Davis SI, Peña C, Xie S, Lauk N, Narváez L et al. Teleportation systems toward a quantum Internet. PRX Quantum. 2020;1(2):20317.

49. Wan KH, Dahlsten O, Kristjánsson H, Gardner R, Kim MS. Quantum generalisation of feedforward neural networks. npj Quantum Inf. 2017:3(1):36

50. Wang XL, Cai XD, Su ZE, Chen MC, Wu D, Li L et al. Quantum teleportation of multiple degrees of freedom of a single photon. Nature. 2015;518(7540):516-9.

51. Yang G, Lian BW, Nie M, Jin J. Bidirectional multi-qubit quantum teleportation in noisy channel aided with weak measurement. Chin Phys B. 2017;26(4):40305.

52. Yeo Y, Skeen A. Time-correlated quantum amplitude-damping channel. Phys Rev A. 2003;67(6):64301.

53. Zhong-Xiao M, Zhan-Jun Z, Yong L. Deterministic secure direct communication by using swapping quantum entanglement and local unitary operations. Chin Phys Lett. 2004:22(1):18-21.

54. Zhou RG, Xu R, lan H. Bidirectional quantum teleportation by using six-qubit cluster state. IEEE Access. 2019:7:44269-75. 